\title{
Influencia de la carbonatación en morteros de cemento Pórtland y ceniza volante
}

\section{Carbonatation Influence on Fly Ash and Portland Cement Mortars}

\author{
P.L. Valdez-Tamez \\ Cuerpo Académico de Tecnología del Concreto \\ Centro de Innovación, Investigación y Desarrollo en Ingeniería y Tecnología (CIIDIT-UANL) \\ E-mail:pvaldez@fic.uanl.mx \\ A. Durán-Herrera \\ Cuerpo Académico de Tecnología del Concreto \\ Centro de Innovación, Investigación y Desarrollo en Ingeniería y Tecnología (CIIDIT-UANL) \\ E-mail:aduran65@yahoo.com.mx \\ G. Fajardo-San Miguel \\ Cuerpo Académico de Tecnología del Concreto \\ Facultad de Ingeniería Civil, Universidad Autónoma de Nuevo León. \\ E-mail:gfajardo@fic.uanl.mx \\ C.A. Juárez-Alvarado \\ Cuerpo Académico de Tecnología del Concreto \\ Centro de Innovación, Investigación y Desarrollo en Ingeniería y Tecnología (CIIDIT-UANL) \\ E-mail:cjuarez@fic.uanl.mx
}

(Recibido: diciembre de 2006; aceptado: enero de 2008)

\section{Resumen}

Se investigó la influencia de la carbonatación en morteros que contienen un $25 \%$ de ceniza volante en sustitución del material cementante. Se fabricaron especímenes cilíndricos de mortero para cuatro relaciones Agua/Cementante (A/C) de 0.35, 0.45, 0.55 y 0.65 . Los morteros con y sin ceniza volante fueron sometidos a un proceso acelerado de carbonatación. Se determinó el peso volumétrico, la absorción, la resistencia a la compresión, la permeabilidad al agua, el pH y la porosimetría por intrusión de mercurio. Por efecto de la potencialidad puzolánica de la ceniza volante, para las cuatro relaciones $\mathrm{A} / \mathrm{C}$, los resultados de la resistencia a la compresión a los 28 días de los morteros con y sin ceniza volante permanecen similares. Los morteros con ceniza volante presentan coeficientes de permeabilidad al agua, a 28 días, similares a los morteros sin ceniza volante. Los resultados de $\mathrm{pH}$ indican que la reducción de la alcalinidad es menor en los morteros que contienen ceniza volante en comparación con aquellos que contienen sólo cemento. Para todos los morteros, los resultados de los análisis porosimétricos indican que la porosidad disminuye por efecto de la carbonatación; asimismo, muestranel predominiodelos macroymesoporos.

Descriptores: Ceniza volante, morteros, carbonatación, porosimetría, alcalinidad.

\section{Abstract}

The influence of carbonation on mortars containing 25\% of fly ash instead of the cementitious materials was studied. Mortar cylinder specimens were fabricated for 4 different W/C ratios: $0.35,0.45,0.55$ and 0.65 . Mortars with and without fly ash were subjected to an accelerated carbonation process. Volumetric weight, water absorption, compressive strength, water permeability, $\mathrm{pH}$ and mercury intrusion porosimetry of the mortar 
DOI: http://dx.doi.org/10.22201/fi.25940732e.2009.10n1.005

Influencia de la carbonatación en morteros de cemento Pórtland y ceniza volante

specimens were determined. Due to the fly ash pozzolanic potential, for all W/C ratios, results of the compressive strength tests at 28 days of the mortars with and without fly ash were similar. Mortars with fly ash presented similar water permeability as mortars without fly ash. PH results showed that alkalinity reduction is lower in mortars with fly ash compared to those containing cement only. In all the mortars, the porosimetric analysis indicated that porosity is reduced due to carbonation. Furthermore, it is showed the predominance of the macro and mesopores.

Keywords: Fly ash, mortars, carbonation, porosimetry, alkalinity.

\section{Introducción}

El concreto es el material de construcción más utilizado en el mundo. En la actualidad, el consumo anual es de aproximadamente 2 toneladas de concreto por habitante. Se estima que se utilizan 5 veces más en peso que el acero, e incluso, en algunos países esta proporción puede llegar a ser de 10 a 1 . No obstante, para producir este material es necesario utilizar cemento Pórtland como aglutinante; sin embargo, por cada tonelada de cemento producida se genera una tonelada de dióxido de carbono $\left(\mathrm{CO}_{2}\right)$ que es emitida a la atmósfera (Aïtcin, 2000), siendo este gas el principal elemento del efecto invernadero que provoca la disminución de la capa de ozono.

En este sentido, existe un claro consenso internacional en que el desarrollo sustentable de las industrias de cemento y concreto puede lograrse con la sustitución parcial de un porcentaje de cemento con materiales cementantes suplementarios, reduciendo con esto la emisión de $\mathrm{CO}_{2}$ a la atmósfera. En la industria del concreto, la ceniza volante (CV) se ha utilizado con éxito, ya que reduce el costo de producción por metro cúbico de este material.

La ceniza volante es un subproducto industrial contaminante que proviene de la industria carboeléctrica y se obtiene al calcinar carbones con impurezas. Su recolección y almacenamiento tienen un valor económico importante que repercute en los costos de producción de la energía eléctrica. Por efecto de su morfología, composición química, mineralógica y tamaño de las partículas, algunas cenizas actúan como un material con una actividad puzolánica significativa (Fraay et al., 1989) i.e. las partículas de ceniza reaccionan con el hidróxido de calcio en presencia de agua para producir un material con características cementantes (Fraay et al., 1989; ACI, 1987). Es así, que su uso resulta en un beneficio económico y en un mejoramiento de ciertas propiedades del concreto en estado fresco (Fraay et al., 1989; ACI, 1987) y endurecido (Fraay et al., 1989; ACI, 1987; Preece et al., 1983; Salta. 1994; Byfors, 1987).
Por otro lado, algunos estudios se han enfocado sobre la importancia de la CV en el mecanismo de corrosión del acero de refuerzo inducido por los cloruros (Montemor, et al., 2000 y 2002; Ampadu et al., 2002), provenientes de sales de deshielo o de un ambiente marino, aspecto que es considerado como la causa principal de deterioro prematuro de las estructuras de concreto reforzado (Bamforth, 1997). Sin embargo, muy pocos trabajos han sido llevados a cabo sobre el papel que juega la CV frente al fenómeno de la carbonatación, que aunque es un fenómeno exclusivo de ambientes urbanos y/o industriales, en algunas regiones puede llegar a manifestarse como el principal problema de degradación por corrosión del acero de refuerzo en estructuras de concreto reforzado.

Por otra parte, es sabido que el $\mathrm{CO}_{2}$ al combinarse con el agua forma el ácido carbónico, el cual baja el $\mathrm{pH}$ a valores cercanos a 6.0 y al entrar en contacto con la superficie del concreto provoca una reacción lenta de neutralización (Metha et al., 2006). Esto ocasiona que el ambiente propicio para la estabilidad termodinámica del acero de refuerzo sea modificado y la protección química que el concreto le confiere al acero desaparezca.

Un concreto permeable puede carbonatarse rápidamente, para esto, basta la pequeña concentración de $\mathrm{CO}_{2}(0.03 \%)$ presente en la atmósfera. Cabe señalar que esta concentración puede incrementarse en lugares específicos, tales como estacionamientos con poca ventilación, túneles, cierto tipo de industrias, laboratorios, etc., lo que acentuará el fenómeno.

Desde el punto de vista químico, la carbonatación del concreto origina la disminución de $\mathrm{pH}$ que ocurre cuando el $\mathrm{CO}_{2}$ del medio ambiente penetra por la red de poros capilares reaccionando con la humedad presente y convirtiendo el hidróxido de calcio (de $\mathrm{pH}$ elevado) a carbonatos, los cuales son neutros, reduciendo por consiguiente, la alcalinidad del concreto.

Las reacciones básicas de la carbonatación en el concreto se manifiestan de la siguiente forma: 
DOI: http://dx.doi.org/10.22201/fi.25940732e.2009.10n1.005

P.L. Valdez-Tamez, A. Durán-Herrera, G. Fajardo-San Miguel y C.A. Juárez-Alvarado

- Los poros de un concreto ordinario, bajo condiciones cíclicas de humedad, contienen:

- Agua $\left(\mathrm{H}_{2} \mathrm{O}\right)$ e

- hidróxido de calcio o cal $\left[\mathrm{Ca}(\mathrm{OH})_{2}\right]$

- Cuando el $\mathrm{CO}_{2}$ del aire entra en contacto con el agua contenida en los poros del concreto, se forma ácido carbónico de acuerdo a:

$\mathrm{CO}_{2}+\mathrm{H}_{2} \mathrm{O} \rightarrow \mathrm{H}_{2} \mathrm{CO}_{3}$

- El ácido carbónico neutraliza el hidróxido de calcio y forma carbonato de calcio sólido, de $\mathrm{pH}$ neutro (Montaini, 1996).

$\mathrm{Ca}(\mathrm{OH})_{2}+\mathrm{H}_{2} \mathrm{CO}_{3} \rightarrow \mathrm{CaCO}_{3}+2 \mathrm{H}_{2} \mathrm{O}$

Alto $\mathrm{pH}$ Bajo $\mathrm{pH}$ pH Neutro

Generalmente, el agua contiene calcio y magnesio en forma de bicarbonatos (como $\mathrm{Ca}\left(\mathrm{HCO}_{3}\right)_{2}$ y $\left.\mathrm{Mg}\left(\mathrm{HCO}_{3}\right)_{2}\right)$, encontrándose el primero en mayor cantidad. Este compuesto aparece en el agua, constituyendo el siguiente equilibrio:

$$
\mathrm{CaCO}_{3}+\mathrm{CO}_{2}+\mathrm{H}_{2} \mathrm{O} \leftrightarrow \mathrm{Ca}\left(\mathrm{HCO}_{3}\right)_{2}
$$

En la ecuación, se constata que es necesaria la presencia de una cantidad libre de $\mathrm{CO}_{2}$ para mantener el equilibrio, que definiremos como $\mathrm{CO}_{2}$ de equilibrio, y que no es perjudicial para el concreto. No obstante, si la cantidad de $\mathrm{CO}_{2}$ libre existente, es mayor que la correspondiente de equilibrio, se rompería el equilibrio y se podría disolver más cal, provocando que el agua se transforme en agresiva.

El mecanismo del ataque de las aguas con $\mathrm{CO}_{2}$ agresivo es el siguiente:

Sobre la superficie del concreto existe $\mathrm{CaCO}_{3}$, como consecuencia de la reacción del $\mathrm{CO}_{2}$ de la atmósfera con el $\mathrm{Ca}(\mathrm{OH})_{2}$, el cual disminuye la permeabilidad del concreto, ya que actúa como sellador debido a su baja solubilidad.

Sin embargo, en presencia de una elevada humedad (producto de la lluvia, condensaciones, etc.) y $\mathrm{CO}_{2}$ agresivo, se efectuará la reacción:

$$
\mathrm{Ca}\left(\mathrm{HCO}_{3}\right)_{2}+\mathrm{Ca}(\mathrm{OH})_{2} \rightarrow 2 \mathrm{CaCO}_{3}+2 \mathrm{H}_{2} \mathrm{O}
$$

El carbonato de calcio obstruye los poros, pero después es atacado nuevamente por el agua agresiva, constituyéndose un proceso dinámico. $\mathrm{El}$ agua con $\mathrm{CO}_{2}$ agresivo puede aparecer en aguas subterráneas, embalses, etc. El agua que contiene más de $20 \mathrm{ppm}$ de $\mathrm{CO}_{2}$ agresivo, puede generar una carbonatación rápida de la pasta de cemento hidratada (ACI, 2001).

El proceso de carbonatación es completamente natural y es afectado también por variables naturales, consecuencia de las condiciones de exposición del concreto. El avance del frente de carbonatación depende principalmente del contenido de humedad relativa (HR), de la permeabilidad del concreto y de la concentración de $\mathrm{CO}_{2}$ disponible en el ambiente.

Ha sido comprobado (Davis et al., 1998) que el mecanismo de reacción de la carbonatación es más rápido cuando la HR está entre el 50 y 75\%. Cuando la HR es menor, no hay suficiente agua en los poros del concreto para que se disuelvan cantidades importantes de hidróxido del calcio. Superior al 75\% de HR, la situación es inversa y los poros pueden llegar a saturarse de agua, en este caso, aunque se permite que el hidróxido de calcio se disuelva libremente, el ingreso del $\mathrm{CO}_{2}$ en los poros saturados es restringido en gran medida. Por otro lado, es bien sabido que la capacidad protectora del recubrimiento depende entre otros factores, del tipo y contenido de cemento, así como de la relación agua/cementante $(\mathrm{A} / \mathrm{C})$ empleada. La adición de CV al concreto puede influir, por tanto, sobre la capacidad protectora de dicho recubrimiento.

En el Departamento de Tecnología del Concreto de la Facultad de Ingeniería Civil de la UANL, se han realizado investigaciones (Rivera et al., 2002, 1997, 1999 y 1998), utilizando CV en el concreto y en algunos casos, empleando altos volúmenes. Los resultados muestran los beneficios físico-químicos y mecánicos que otorga la $\mathrm{CV}$ al concreto. El presente trabajo tiene como objetivo estudiar los efectos de la utilización de CV tipo "F" de MICARE (Minera Carbonífera de Río Escondido, Coahuila, México) en morteros fabricados con cemento Pórtland ordinario (CPO) expuestos a una atmósfera rica en $\mathrm{CO}_{2}$. Para evaluar lo anterior, se realizaron mediciones de porosimetría y del $\mathrm{pH}$ de la matriz en morteros no carbonatados (MNC) y en morteros carbonata$\operatorname{dos}(\mathrm{MN})$.

\section{Desarrollo experimental}

\section{Materiales}

Para realizar las mezclas de morteros se utilizó CPO producido por la empresa CEMEX y CV tipo "F" de MICARE. Las propiedades físicas, químicas y mecánicas de estos materiales se presentan en las tablas 1 y 3 . La CV fue utilizada 
como sustitución parcial del 25\% del cemento, porcentaje en masa. Esta ceniza cumple satisfactoriamente con la cantidad mínima de $\mathrm{SiO}_{2}+\mathrm{Al}_{2} \mathrm{O}_{3}+\mathrm{Fe}_{2} \mathrm{O}_{3}$ establecida por la especificación ASTM C 618-05, ya que alcanza una concentración de $92.5 \%$ contra un mínimo del $70.0 \%$ que establece la norma, así mismo, cumple con los límites del índice de actividad a la resistencia (IAR $\geq 75 \%$ ).

Se utilizó agregado fino calizo de la región, cuyas propiedades físicas y distribución granulométrica son presentadas en las tablas 1 y 2 , respectivamente. Con el objetivo de eliminar la influencia de la distribución de partículas del agregado fino, se utilizó una graduación intermedia entre los límites superior e inferior especificados por la ASTM C 33-03.

\section{Fabricación de especímenes}

Se utilizó agregado fino con un tamaño máximo nominal de $4.8 \mathrm{~mm}$ (\#4) con relaciones $\mathrm{A} / \mathrm{C}$ de $0.35,0.45$ 0.55 y 0.65 y un mini-revenimiento (cono truncado de $10 \mathrm{~cm}$ de diámetro en la base, $5 \mathrm{~cm}$ de diámetro en la parte superior y $15 \mathrm{~cm}$ de altura) de $5 \mathrm{~cm} \pm 1 \mathrm{~cm}$.

Lo anterior dio como resultado un total de ocho mezclas de mortero ( 4 sin CV y 4 con CV). Las proporciones de los materiales utilizados se muestran en la tabla 4.

A los morteros se les determinó el contenido de aire atrapado en la mezcla en estado fresco utilizando el método descrito en ASTM C 231-04. Para cada una de las mezclas se fabricaron 10 especímenes cilíndricos de 10

Tabla 1. Propiedades físicas de los materiales

\begin{tabular}{|c|c|c|c|c|}
\hline \multicolumn{2}{|c|}{ Propiedades } & $\mathrm{CPO}$ & CV & Agregado fino \\
\hline \multicolumn{2}{|c|}{ Peso volumétrico seco suelto, $\mathrm{kN} / \mathrm{m}^{3}$} & 12.26 & 8.06 & 15.30 \\
\hline \multicolumn{2}{|c|}{ Densidad } & 3.09 & 2.34 & 2.64 \\
\hline \multicolumn{2}{|c|}{ Superficie específica (Blaine), $\mathrm{m}^{2} / \mathrm{kg}$} & 360 & 440 & - \\
\hline \multicolumn{2}{|c|}{ Absorción, \% } & - & 23.9 & 0.9 \\
\hline \multicolumn{2}{|c|}{ Fineza (malla \#325), \% retenido } & - & 5 & - \\
\hline \multirow{2}{*}{$\begin{array}{l}\text { Tiempos de fraguado } \\
\text { Vicat, minutos }\end{array}$} & Inicial & 96 & - & - \\
\hline & Final & 171 & - & - \\
\hline \multirow{5}{*}{$\begin{array}{l}\text { Resistencia a la } \\
\text { compresión, } \mathrm{MPa}\end{array}$} & $1 \mathrm{~d}$ & 6.5 & - & - \\
\hline & $3 d$ & 19.6 & - & - \\
\hline & $7 \mathrm{~d}$ & 20.8 & - & - \\
\hline & $28 \mathrm{~d}$ & 28.4 & - & - \\
\hline & $56 \mathrm{~d}$ & 35.9 & - & - \\
\hline \multirow{2}{*}{$\begin{array}{l}\text { Índice de actividad } \\
\text { a la resistencia, \% }\end{array}$} & $7 d$ & - & 76 & - \\
\hline & $28 \mathrm{~d}$ & - & 84 & - \\
\hline
\end{tabular}

Tabla 2. Granulometría del agregado fino

\begin{tabular}{cccccccc}
\hline \multicolumn{7}{c}{ Análisis granulométrico } \\
\hline Tamaño de malla, mm & $0.15(\# 100)$ & $0.30(\# 50)$ & $0.60(\# 30)$ & $1.18(\# 16)$ & $2.36(\# 8)$ & $4.75(\# 4)$ & $9.50(3 / 8 ")$ \\
$\%$ que pasa & 6 & 20 & 43 & 68 & 90 & 98 & 100 \\
\hline
\end{tabular}


DOI: http://dx.doi.org/10.22201/fi.25940732e.2009.10n1.005

P.L. Valdez-Tamez, A. Durán-Herrera, G. Fajardo-San Miguel y C.A. Juárez-Alvarado

Tabla 3. Composición química de cementantes

\begin{tabular}{|c|c|c|c|c|c|c|c|c|c|c|c|c|}
\hline \multirow{2}{*}{ Material } & \multicolumn{12}{|c|}{ Composición química \% } \\
\hline & $\mathrm{SiO}_{2}$ & $\mathrm{Al}_{2} \mathrm{O}_{3}$ & $\mathrm{Fe}_{2} \mathrm{O}_{3}$ & $\mathrm{CaO}$ & $\mathrm{MgO}$ & $\mathrm{SO}_{3}$ & $\mathrm{Na}_{2} \mathrm{O}$ & $\mathrm{K}_{2} \mathrm{O}$ & $\mathrm{TiO}_{2}$ & $\mathrm{P}_{2} \mathrm{O}_{5}$ & $\mathrm{Mn}_{2} \mathrm{O}_{3}$ & P.I. \\
\hline $\mathrm{CPO}$ & 20.50 & 4.70 & 2.10 & 64.20 & 1.70 & 3.10 & 0.17 & 0.77 & 0.19 & 0.09 & 0.04 & 2.40 \\
\hline \multirow[t]{2}{*}{ CV } & 60.10 & 27.80 & 4.60 & 1.85 & 0.65 & 0.90 & 0.55 & 0.80 & 0.95 & 0.25 & 0.00 & 1.55 \\
\hline & & & & & \multicolumn{8}{|c|}{ Compuestos potenciales } \\
\hline Material & $\begin{array}{c}\mathrm{SiO}_{2}+ \\
\mathrm{Al}_{2} \mathrm{O}_{3}+ \\
\mathrm{Fe}_{2} \mathrm{O}_{3}\end{array}$ & $\begin{array}{l}\mathrm{CaO} \\
\text { libre }\end{array}$ & $\begin{array}{l}\text { Residuo } \\
\text { insoluble }\end{array}$ & $\begin{array}{c}\text { Álcalis } \\
\text { totales } \\
\mathrm{Na}_{2} \mathrm{O}\end{array}$ & \multicolumn{2}{|c|}{$\mathrm{C}_{3} \mathrm{~S}$} & \multicolumn{2}{|c|}{$\mathrm{C}_{2} \mathrm{~S}$} & \multicolumn{2}{|c|}{$\mathrm{C}_{3} \mathrm{~A}$} & \multicolumn{2}{|c|}{$\mathrm{C}_{4} \mathrm{AF}$} \\
\hline $\mathrm{CPO}$ & 27.30 & 0.70 & 0.33 & 0.68 & \multicolumn{2}{|c|}{61.80} & \multicolumn{2}{|c|}{12.50} & \multicolumn{2}{|c|}{8.90} & \multicolumn{2}{|c|}{6.40} \\
\hline $\mathrm{CV}$ & 92.50 & - & - & 1.08 & \multicolumn{2}{|c|}{ - } & & & \multicolumn{2}{|c|}{ - } & \multicolumn{2}{|c|}{ - } \\
\hline
\end{tabular}

P.I. = Pérdida por ignición

Tabla 4. Proporciones de los morteros

\begin{tabular}{|c|c|c|c|c|c|c|c|c|c|}
\hline \multirow{3}{*}{\multicolumn{2}{|c|}{ Material }} & \multicolumn{8}{|c|}{ Proporciones de materiales, $\mathrm{kg} / \mathrm{m}^{3}$} \\
\hline & & \multicolumn{4}{|c|}{$\mathrm{A} / \mathrm{C}$, morteros $\sin \mathrm{CV}$} & \multicolumn{4}{|c|}{$\mathrm{A} / \mathrm{C}$, morteros con $\mathrm{CV}$} \\
\hline & & 0.35 & 0.45 & 0.55 & 0.65 & 0.35 & 0.45 & 0.55 & 0.65 \\
\hline \multirow{2}{*}{ Agua } & Reacción & 280.0 & 247.0 & 244.0 & 234.0 & 280.0 & 247.0 & 244.0 & 234.0 \\
\hline & Absorción & 10.2 & 12.3 & 13.2 & 14.3 & 10.1 & 12.9 & 13.8 & 14.8 \\
\hline \multicolumn{2}{|c|}{$\mathrm{CPO}$} & 800.0 & 549.0 & 444.0 & 360.0 & 600.0 & 412.0 & 333.0 & 270.0 \\
\hline \multicolumn{2}{|c|}{ CV } & - & - & - & - & 200.0 & 137.0 & 111.0 & 90.0 \\
\hline \multicolumn{2}{|c|}{ Cementante total } & 800.0 & 549.0 & 444.0 & 360.0 & 800.0 & 549.0 & 444.0 & 360.0 \\
\hline \multicolumn{2}{|c|}{$\mathrm{AF}$} & 1138.0 & 1370.0 & 1468.0 & 1592.0 & 1125.0 & 1439.0 & 1537.0 & 1648.0 \\
\hline \multicolumn{2}{|c|}{$\%$ de pasta } & 48.7 & 36.7 & 31.9 & 27.2 & 49.0 & 35.6 & 30.9 & 26.5 \\
\hline
\end{tabular}

$\mathrm{cm}$ de diámetro por $20 \mathrm{~cm}$ de altura, utilizando una revolvedora convencional de 100 litros de capacidad. Los especímenes fueron mantenidos en sus moldes por 24 horas en un ambiente con temperatura de laboratorio y protegidos del intercambio de humedad. Posteriormente fueron desmoldados y curados en un cuarto a $23 \pm 1$ ${ }^{\circ} \mathrm{C}$ y con una HR superior al $95 \%$, en donde permanecieron por un periodo de 28 días.

Después del periodo de curado y justo antes de someterlos al proceso de carbonatación se hicieron pruebas de resistencia a la compresión de acuerdo a la norma ASTM C 39-05, de absorción de acuerdo a la norma ASTM C 642-05, de porosimetría y de permeabilidad al agua. Los resultados de estas pruebas, para las mezclas fabricadas con y sin ceniza son presentados en las tablas 5 y 6 .
También se realizaron mediciones de la alcalinidad de la matriz de mortero, a través de la medida del $\mathrm{pH}$.

\section{Acondicionamiento de especímenes, prepara- ción de muestras y exposición}

Para cada relación $\mathrm{A} / \mathrm{C}$, uno de los especimenes cilíndricos fue seccionado transversalmente hasta obtener 4 muestras de aproximadamente $4.5 \mathrm{~cm}$ de altura, utilizando para esto una cortadora de disco diamantado. Posteriormente, cada sección fue sellada lateralmente con membrana polimérica. Para obtener el coeficiente de permeabilidad, estas muestras fueron sometidas a una presión hidrostática de 30 bares por 24 horas.

Los análisis porosimétricos se realizaron antes y después del periodo de exposición. Para ello, otro espécimen 
de cada relación $\mathrm{A} / \mathrm{C}$ fue cortado en secciones y triturado, seleccionando la muestra con tamaños de partículas entre 2.5 y $5 \mathrm{~mm}$. Estas muestras se colocaron en un desecador hasta peso constante, posteriormente se realizaron las pruebas empleando un Porosímetro por intrusión de mercurio marca Carlo Erba con capacidad máxima de 2000 bares.

El pH de la matriz de mortero fue obtenido antes y después de la exposición. Para ello, se pulverizaron muestras representativas de los diferentes especímenes hasta un tamaño de partícula de $0.30 \mathrm{~mm}$ (\#50). La muestra en polvo fue disuelta en agua destilada en una relación 1:5 en peso. Posteriormente se determinó el pH de las muestras utilizando un potenciómetro digital.

Para inducir y acelerar la cinética del proceso de carbonatación en los especímenes, se utilizó una cámara de carbonatación en la cual se inyectó gas con una concentración de $\mathrm{CO}_{2}$ al $5 \%$, poco más de 150 veces la concentración normal del aire atmosférico. La cámara fue provista de un ventilador en el interior para forzar la circulación del gas. Con el propósito de asegurar una concentración constante de $\mathrm{CO}_{2}$ en el interior, la cámara de carbonatación fue provista de una ventila para retirar el aire atmosférico al momento de la inyección de $\mathrm{CO}_{2}$.

El periodo de carbonatación acelerada después del curado, fue de cinco meses, tiempo en el que se carbonataron completamente todas las muestras de mortero con y sin ceniza, y para las diferentes relaciones A/C. Lo anterior fue verificado al utilizar frecuentemente el método de la fenolftaleina.

\section{Resultados y discusión}

En la tabla 5, se muestran los resultados de las propiedades del mortero en estado fresco y endurecido, tales como mini-revenimiento, aire atrapado, absorción, peso volumétrico y resistencia a la compresión. Los resultados de las pruebas de aire atrapado en morteros en estado fresco reflejan el efecto densificador de la CV. El porcentaje de absorción de agua aumenta conforme se incrementa la relación $\mathrm{A} / \mathrm{C}$ y al comparar los valores de los morteros con ceniza y sin ceniza, se presenta en promedio un $40 \%$ más de absorción para los morteros sin ceniza, lo anterior se atribuye al efecto densificador de la CV y su acción puzolánica.

Cabe recordar que los morteros con CV fueron fabricados sólo con el 75\% de CPO, con relación a los morteros sin CV. Por otro lado, se puede observar que el peso volumétrico para las cuatro relaciones $\mathrm{A} / \mathrm{C}$ y para los especímenes fabricados con y sin ceniza disminuyó al aumentar la relación A/C.
Con relación a la resistencia a la compresión a 28 días, se presenta en promedio una reducción del $10 \%$ en los especímenes fabricados con ceniza y para las cuatro relaciones $\mathrm{A} / \mathrm{C}$, comparados con aquellos fabricados sin ceniza. En este sentido, un reporte del Comité ACI 226 (1987) menciona que una vez que la ganancia de la resistencia mecánica del cemento Pórtland ha disminuido, la actividad puzolánica de la CV continua desarrollándose, contribuyendo a incrementar la resistencia mecánica a edades posteriores, si el concreto es conservado en condiciones de elevada humedad. Otro estudio efectuado por Lane et al. (1982), utilizando resistencias de referencia obtenidas a 28 días, reportaron incrementos de hasta un $50 \%$ en la resistencia obtenida a un año para concretos con CV comparado con solo un $30 \%$ con concretos fabricados sin ceniza. Por lo tanto, morteros con CV que presentan resistencias bajas o equivalentes a edades tempranas, pueden presentar resistencias mecánicas mayores a edades posteriores que aquellos en los cuales no se incluya CV.

En la tabla 6, se presentan los resultados de las pruebas de permeabilidad al agua obtenidos después de 28 días de curado (MNC). En ella se puede observar que para ambas series (con y sin ceniza) el coeficiente de permeabilidad $(K)$ o de Darcy se incrementa al aumentar la relación $\mathrm{A} / \mathrm{C}$. Este comportamiento resulta lógico, ya que el concreto es menos denso. Sin embargo, al comparar los resultados obtenidos en las dos series y para las relaciones $\mathrm{A} / \mathrm{C}$ de 0.55 y 0.65 , la utilización de ceniza origina en promedio una disminución del $10 \%$ en el coeficiente de permeabilidad, debido a la discontinuidad del sistema poroso. Se estima que este porcentaje se incrementará con el desarrollo de la acción puzolánica (Malhotra et al., 2005).

En la tabla 6 se presentan los resultados de los análisis de $\mathrm{pH}$, para los MNC y MC con y sin ceniza. En ella se puede observar que, para los $\mathrm{MC}$, el pH de la matriz tiende a disminuir conforme se incrementa la relación A/C. Lo anterior, debido a las diferencias de los consumos de cemento, ya que en el caso de morteros con relaciones A/C altas (0.65) el consumo de cemento es menor, razón por la cual existiría una menor cantidad de hidróxidos generada. Asimismo, las relaciones $\mathrm{A} / \mathrm{C}$ altas permiten una estructura de la red de poros más abierta, facilitando con ello el ingreso y la difusión del $\mathrm{CO}_{2}$, propiciando el avance de la carbonatación. Sin embargo, al comparar los valores de $\mathrm{pH}$ obtenidos al final del periodo de exposición, para los morteros con ceniza, el pH alcanza valores mayores para casi todas las relaciones A/C, con excepción de los morteros con relación $\mathrm{A} / \mathrm{C}$ de 0.35. Lo anterior podría indicar que, para un mismo 
tiempo de exposición, la disminución de la alcalinidad es menos significativa en los especímenes que contienen ceniza, comparados con aquellos especímenes fabricados sólo con CPO.

En la figura 1 se muestran los resultados de la porosidad total con función en la relación A/C para los especímenes con y sin ceniza, antes y después del periodo de carbonatación. En la tabla 6 se muestran los resultados de las pruebas de porosidad total de los especímenes en función de la relación A/C, fabricados con y sin ceniza volante.

Por un lado, se aprecia que, al final del periodo de curado, la porosidad total de los especímenes fabricados con ceniza se incrementa, variando de un $15.6 \%$, para la

Tabla 5. Propiedades físicas de los morteros

\begin{tabular}{|c|c|c|c|c|c|c|c|c|}
\hline \multirow{2}{*}{ Propiedades } & \multicolumn{4}{|c|}{$\mathrm{A} / \mathrm{C}$, mortero $\sin \mathrm{CV}$} & \multicolumn{4}{|c|}{$\mathrm{A} / \mathrm{C}$, mortero con $\mathrm{CV}$} \\
\hline & 0.35 & 0.45 & 0.55 & 0.65 & 0.35 & 0.45 & 0.55 & 0.65 \\
\hline & \multicolumn{8}{|c|}{ Estado fresco } \\
\hline Mini revenimiento & 4.5 & 4.5 & 4.5 & 4.5 & 5.0 & 5.0 & 5.0 & 5.0 \\
\hline \multirow[t]{2}{*}{ Aire atrapado \% } & 3.0 & 5.6 & 5.6 & 4.6 & 2.5 & 3.7 & 4.7 & 4.3 \\
\hline & \multicolumn{8}{|c|}{ Estado endurecido } \\
\hline Absorción \% & 8.2 & 8.3 & 8.6 & 9.6 & 5.2 & 6.2 & 6.5 & 6.7 \\
\hline $\begin{array}{l}\text { Peso volumétrico } \\
\text { saturado } \\
\text { superficialmente } \\
\text { seco, } \mathrm{kN} / \mathrm{m}^{3}\end{array}$ & 22.6 & 22.4 & 22.1 & 21.9 & 21.6 & 21.4 & 21.3 & 21.1 \\
\hline $\begin{array}{c}\text { Resistencia a la } \\
\text { compresión a los } 28 \\
\text { días, } \mathrm{MPa}\end{array}$ & 62.9 & 45.4 & 39.4 & 29.7 & 58.4 & 40.6 & 34.7 & 27.9 \\
\hline
\end{tabular}

Tabla 6. Permeabilidad, $\mathrm{pH}$ y porosidad de los morteros

\begin{tabular}{ccccccccc}
\hline \multirow{2}{*}{ Material } & \multicolumn{9}{c}{$\mathrm{A} / \mathrm{C}$, mortero sin CV } & A/C, mortero con CV \\
\cline { 2 - 8 } & 0.35 & 0.45 & 0.55 & 0.65 & 0.35 & 0.45 & 0.55 & 0.65 \\
\hline MNC & 0.07 & 0.75 & 1.31 & 10.10 & 0.08 & 0.73 & 1.20 & 9.00 \\
\hline & & \multicolumn{7}{c}{ Coeficiente de permeabilidad al agua $(\mathrm{K}, \mathrm{cm} / \mathrm{s}) 10^{-10}$} \\
\hline $\begin{array}{c}\text { Consumo de cemento } \\
\mathrm{kg} / \mathrm{m}^{3}\end{array}$ & 800.0 & 549.0 & 444.0 & 360.0 & 600.0 & 412.0 & 333.0 & 270.0 \\
MNC & 12.6 & 12.5 & 12.4 & 12.1 & 12.7 & 12.7 & 12.6 & 12.7 \\
MC & 11.3 & 9.0 & 8.5 & 8.1 & 11.3 & 10.3 & 9.9 & 8.7 \\
\hline MNC & 13.2 & 14.5 & 14.4 & 17.4 & 15.6 & 17.4 & 20.1 & 24.3 \\
MC & 5.8 & 8.5 & 10.8 & 14.3 & 12.1 & 13.3 & 13.9 & 16.6 \\
Variación & 7.4 & 6.0 & 3.6 & 3.1 & 3.5 & 4.1 & 6.2 & 7.7 \\
\hline
\end{tabular}




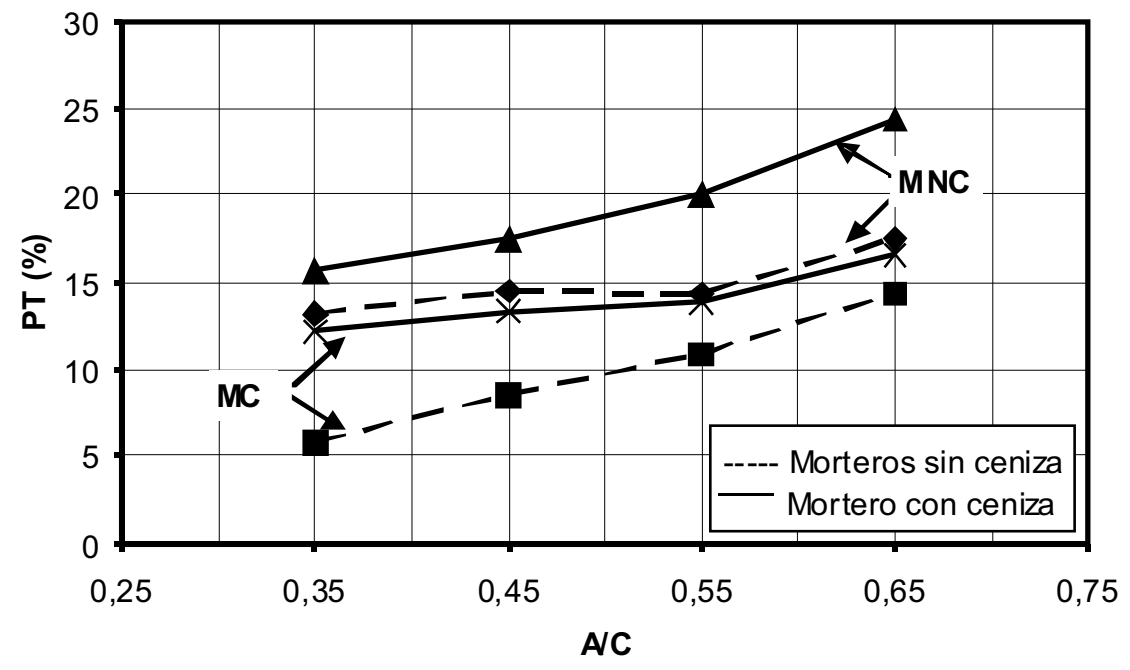

Figura 1. Relación A/C vs. Porosidad Total (PT) de morteros no carbonatados (MNC) y carbonatados (MC)

relación $\mathrm{A} / \mathrm{C}$ de 0.35 a un 24.3 \% para la relación $\mathrm{A} / \mathrm{C}$ de 0.65. Después del periodo de exposición en los MC la porosidad total de estos mismos especímenes se reduce a 12.1 y $16.6 \%$, respectivamente. Para el caso de los morteros sin ceniza y sin carbonatar, se presenta un incremento en la porosidad total, variando de un $13.2 \%$ para la relación $\mathrm{A} / \mathrm{C}$ de 0.35 a un $17.4 \%$ para la relación $\mathrm{A} / \mathrm{C}$ de 0.65 ; sin embargo, al carbonatarse, estos valores son reducidos a 5.8 y $14.3 \%$, respectivamente. Este comportamiento, sería atribuido al proceso por el cual el $\mathrm{CO}_{2}$ reacciona con el $\mathrm{Ca}(\mathrm{OH})_{2}$ y forma $\mathrm{CaCO}_{3}$ que actúa como sellador y es poco soluble, reduciendo por consiguiente la porosidad.

Con relación a la variación de la porosidad por el efecto de la carbonatación, podemos observar que existe una reducción para los morteros sin ceniza, dicha reducción varió de 7.4 a $3.1 \%$ para las relaciones $\mathrm{A} / \mathrm{C}$ de 0.35 a 0.65 respectivamente. Para los morteros con ceniza, varía de un 3.5 a un $7.7 \%$ al ir aumentando la relación A/C.
La tabla 7 muestra la distribución por rangos del radio promedio de los poros, obtenidos por la técnica de porosimetría por intrusión de mercurio, al final del periodo de curado y posterior al periodo de exposición al $\mathrm{CO}_{2}$.

Se puede observar que tanto para MC y MNC, independientemente de la formulación del mortero empleado, existe un predominio de macro y mesoporos. A medida que se incrementa la relación $\mathrm{A} / \mathrm{C}$ disminuyen los mesoporos y aumentan los macroporos. Lo anterior probablemente debido a que la estructura interna de estos poros es más débil para relaciones $\mathrm{A} / \mathrm{C}$ mayores.

Por consiguiente, al carbonatarse la matriz que los contiene, disminuye la cantidad de mesoporos y se incrementan los macroporos y ultramacroporos. Lo anterior para los morteros con y sin ceniza y todas las relaciones A/C, lo cual puede justificarse por la formación de carbonatos en la región de los mesoporos. 
DOI: http://dx.doi.org/10.22201/fi.25940732e.2009.10n1.005

P.L. Valdez-Tamez, A. Durán-Herrera, G. Fajardo-San Miguel y C.A. Juárez-Alvarado

Tabla 7. Distribución por rangos del radio del poro

\begin{tabular}{|c|c|c|c|c|c|}
\hline \multirow{3}{*}{ Condición } & \multirow{3}{*}{$\mathrm{A} / \mathrm{C}$} & \multirow{3}{*}{ Porosidad Total \% } & \multicolumn{3}{|c|}{ Distribución del radio del poro \% } \\
\hline & & & Ultramacroporos & Macroporos & Mesoporos \\
\hline & & & $r>75,000 \AA$ & $300 \leq \mathrm{r} \leq 75,000 \AA$ & $37 \leq \mathrm{r}<300 \AA$ \\
\hline \multicolumn{6}{|c|}{ Mortero sin CV } \\
\hline \multirow{4}{*}{ MNC } & 0.35 & 13.2 & 4 & 18 & 78 \\
\hline & 0.45 & 14.5 & 3 & 35 & 62 \\
\hline & 0.55 & 14.4 & 4 & 41 & 56 \\
\hline & 0.65 & 17.4 & 3 & 52 & 45 \\
\hline \multirow{4}{*}{ MC } & 0.35 & 5.8 & 7 & 30 & 63 \\
\hline & 0.45 & 8.5 & 7 & 38 & 55 \\
\hline & 0.55 & 10.8 & 9 & 42 & 49 \\
\hline & 0.65 & 14.3 & 5 & 57 & 38 \\
\hline \multicolumn{6}{|c|}{ Mortero con CV } \\
\hline \multirow{5}{*}{ MNC } & 0.35 & 15.6 & 3 & 18 & 79 \\
\hline & 0.45 & 17.4 & 3 & 38 & 59 \\
\hline & 0.55 & 20.1 & 2 & 46 & 52 \\
\hline & 0.65 & 24.3 & 2 & 50 & 48 \\
\hline & 0.35 & 12.1 & 5 & 30 & 65 \\
\hline \multirow{3}{*}{$\mathrm{MC}$} & 0.45 & 13.3 & 6 & 38 & 56 \\
\hline & 0.55 & 13.9 & 6 & 51 & 43 \\
\hline & 0.65 & 16.6 & 6 & 58 & 36 \\
\hline
\end{tabular}

\section{Conclusiones}

Al remplazar el 25\% del CPO por CV tipo "F" en la producción de morteros curados de forma estándar por 28 días, se observa que la resistencia mecánica a la compresión es similar a los morteros sin ceniza.

Los morteros con ceniza manifiestan un incremento en la porosidad total para las cuatro relaciones $\mathrm{A} / \mathrm{C}$ con relación a los morteros sin ceniza, especialmente aquellos con alta relación A/C.

Los morteros con y sin ceniza presentan coeficientes de permeabilidad al agua del mismo orden de magnitud.

En los morteros con y sin CV, el efecto de la carbonatación produjo una reducción en la porosidad total.

La disminución de alcalinidad debido a la carbonatación es menor en los morteros que contienen ceniza volante, comparados con aquellos que no contienen ceniza.
El conjunto de resultados obtenidos a los 28 días de curado, indican que el uso de este tipo de puzolanas requiere un mayor tiempo de reactividad para lograr desarrollar al máximo su actividad puzolánica.

\section{Referencias}

Aïtcin P.C. Cement and Concrete Development from an Environmental Perspective, Concrete Technology for a Sustainable Development in the 21st Century. Edited by Odd Gjørv and Koji Sakai. 2000. Pp. 210.

Fraay A., Bijen J.M. and Haan Y.M. The Reaction of Fly Ah in Concrete a Critical Examination. Cement and Concrete Research, 19:235-246. 1989.

ACI. Use of Fly Ash in Concrete. ACI Committee 226 Report. ACI Materials Journal, septiembre-octubre:81.1987. 
Preece C.M., Gonvold F.O., Frolund T. Corrosion of Reinforcement in Concrete Constructions. In: Crane A.P. ed. London, U.K., 1983, pp. 393.

Salta M.M. Corrosion and Corrosion Protection of Steel in Concrete. In: Swamy R.N. ed. Sheffield, U.K., 1994, pp. 793.

Byfors K. Influence of Silica Fume and Fly Ash on Chloride Diffusion and $\mathrm{pH}$ Values in Cement Paste. Cement and Concrete Research, 17:115-130. 1987.

Montemor M.F., Simoes A.M.P., Salta M.M. Effect of Fly Ash on Concrete Reinforcement Corrosion Studied by EIS. Cement and Concrete Composites. 22: 175-185. 2000.

Montemor M.F., Cunha M.P., Ferreira M.G., Simoes A.M. Corrosion Behaviour of Rebars in Fly Ash Mortar Exposed to Carbon Dioxide and Chlorides. Cement and Concrete Composites. 24: 45-53. 2002.

Ampadu K.O., Torii K. Chloride Ingress and Steel Corrosion in Cement Mortars Incorporating Low- Quantity Fly Ashes. Cement and Concrete Research. 32:893-901. 2002.

Bamforth P., Price W.F., Emmerson M. International Review of Chloride Ingress into Structural Concrete, Contractor Report 354. Edinburgh, Scotland. Transport Research Laboratory. 1997.

Metha K., Monteiro-Paulo J.M. Concrete. Third Edition. Mc Graw Hill. 2006. Pp. 155.

Montaini R. Concretes Forgotten Enemy. Concrete Repair Digest. December 1995/January 1996. Pp. 392.

ACI Committee 201. Guide to Durable Concrete. ACI Manual of Concrete Practice. Part 1: 201.2 R-11-201.2 R-12. 2004.
Davis H., Rothwell G.W. The Effectiveness of Surface Coating in Reducing Carbonation of Reinforced Concrete. BRE Information. 1998.

Rivera R., Rivera J.M. Effect of Curing in a High Temperature Environment on Compressive Strength of Concrete Incorporating Different Complementary Cementitious Materials. Proceedings, ACI. Fifth International Conference, Cancun, México, SP-209, 2002, pp. 1-28.

Rivera R., Dávila R., Durán A. High Strength High Performance Concrete Using Low and High Volumes of Fly Ash and High-Range Water-Reducing Admixture. Fifth CANMET/ ACI International Conference on Superplasticizers and Other Chemical Admixtures in Concrete, Rome, Italy, 1997, pp. 271-286.

Rivera R., Dávila R., Durán A. High Strength High Performance Concrete Using Fly Ash and Superplasticizer Admixture. Proceedings. Second CANMET/ACI International Conference, Gramado, RS, Brazil, SP-186, 1999, pp. 341-356.

Rivera R., Dávila R., Durán A. High Performance Concrete Using Fly Ash and Superplasticizer. International Symposium on High-Performance and Reactive Powder Concretes, Université de Sherbrooke, Québec, Canada, 1998, pp. 301-315.

Lane R.O., Best J.F. Propierties and Use of Fly Ash in Portland Cement Concrete. Concrete International: Desing \& Construction, 4(7):81-82. July 1982.

Malhotra V.M., Metha P.K. High Volume Fly Ash Concrete. Second Edition.CANMET. 2005. Pp 55. 
DOI: http://dx.doi.org/10.22201/fi.25940732e.2009.10n1.005

P.L. Valdez-Tamez, A. Durán-Herrera, G. Fajardo-San Miguel y C.A. Juárez-Alvarado

\section{Semblanza de los autores}

Pedro Leobardo Valdez-Tamez. Es ingeniero civil, maestro en ciencias con especialidad en ingeniería ambiental, doctor en ingeniería de materiales por la Facultad de Ingeniería Mecánica y Eléctrica, UANL. Es profesor de tiempo completo en la Universidad Autónoma de Nuevo León (UANL). Realizó estancias de investigación en el 2005 y 2006 en la Universidad Politécnica de Cataluña, España. Subdirector de estudios de posgrado e investigación de la Facultad de Ingeniería Civil (FIC) de la UANL y miembro del SNI con Perfil deseable (PROMEP). Profesor visitante del Centro Interdisciplinario de Investigación para el Desarrollo Integral Regional del Instituto Politécnico Nacional, Unidad Oaxaca. Tiene más de 10 años de experiencia en docencia tanto a nivel licenciatura como en posgrado y más de 16 años en el área de la tecnología del concreto. Experto académico representante de la FIC ante el Consorcio de Universidades Mexicanas (CuMex). Presidente de la Sección Noreste de México del American Concrete Institute (ACI) y examinador del programa de certificación de Técnicos en Pruebas de Concreto del ACI. Ha dirigido más de 12 tesis (licenciatura, maestría y doctorado) y ha producido más de 20 artículos de investigación.

Alejandro Durán-Herrera. Es ingeniero civil, maestro en ciencias con orientación en ingeniería ambiental y doctor en ingeniería de materiales por la UANL. Es profesor investigador en la Facultad de Ingeniería Civil de la Universidad Autónoma de Nuevo León (UANL). Jefe del Departamento de Tecnología del Concreto del Instituto de Ingeniería Civil. Es miembro del Sistema Nacional de Investigadores (SNI) y recibió el reconocimiento por la SEP como profesor con perfil deseable (PROMEP). Miembro Fellow y Young Member Award 2004 del American Concrete Institute (ACI) y miembro de varios de sus comités. Sus temas de investigación son: Ingredientes para concreto, concretos de alto comportamiento, uso de ceniza volante en el concreto, durabilidad del concreto y curado del concreto. Actualmente es profesor de tiempo completo de la Universidad Autónoma de Nuevo León (UANL).

Gerardo Fajardo-San Miguel. Es ingeniero mecánico, maestro en ciencias con especialidad en ingeniería mecánica por el Instituto Tecnológico de Veracruz y doctor en ingeniería civil de la Université Paul Sabatier de Toulouse, Francia. Es especialista en durabilidad de obras de concreto y deterioro de materiales de ingeniería, candidato a Investigador Nacional del SNI y Perfil deseable (PROMEP) que avalan más de 5 años de experiencia en docencia tanto a nivel licenciatura como en posgrado y más de 10 años de experiencia en el área de la durabilidad de obras de concreto evidenciados por más de 20 artículos de investigación publicados. Actualmente es profesor de tiempo completo en la Universidad autónoma de Nuevo León.

César Antonio Juárez-Alvarado. Es ingeniero civil (1991), por la Facultad de Ingeniería Civil de la UANL. Maestro en ciencias con especialidad en ingeniería estructural (1998), en la Facultad de Ingeniería Civil de la UANL y doctor en ingeniería de materiales (2002), con la calificación MAGNA CUM LAURO, en el programa doctoral de ingeniería de materiales, de la Facultad de Ingeniería Mecánica y Eléctrica de la UANL. Es miembro del SNI en el área de ingeniería y tecnología en la disciplina de ingeniería de materiales. Fue reconocido por la SEP como profesor de tiempo completo con perfil deseable. Ingeniero especialista en estructuras, consultor en análisis y diseño estructural, así como en el uso de materiales de construcción. De 1993 a 2002, ha sido profesor por asignatura en la Facultad de Ingeniería Civil de la UANL. A partir de agosto del 2002 a la fecha es profesor investigador de tiempo completo de la Facultad de Ingeniería Civil de la UANL, impartiendo docencia en licenciatura y posgrado. Ha dirigido tesis de licenciatura y de maestría, publicado artículos en revistas internacionales indexadas y en revistas nacionales con estricto arbitraje, ha realizado proyectos de investigación para la SEP, CONACYT, etc. Ha sido ganador del premio de investigación de la UANL 2002 y del premio por la dirección de la mejor tesis de licenciatura 2005. 